\title{
Distributed Sensor Local Linear Fusion Detection of Weak Pulse Signal in Chaotic Background
}

\author{
Liyun Su $(\mathbb{D}$, Meini Li $\mathbb{D}$, Shengli Zhao $\mathbb{D}$, and Ting Xie \\ School of Science, Chongqing University of Technology, Chongqing 400054, China \\ Correspondence should be addressed to Liyun Su; cloudhopping@163.com
}

Received 24 November 2020; Revised 19 January 2021; Accepted 25 January 2021; Published 10 February 2021

Academic Editor: Giovanni Diraco

Copyright @ 2021 Liyun Su et al. This is an open access article distributed under the Creative Commons Attribution License, which permits unrestricted use, distribution, and reproduction in any medium, provided the original work is properly cited.

\begin{abstract}
This paper combines the distributed sensor fusion system with the signal detection under chaotic noise to realize the distributed sensor fusion detection from chaotic background. First, based on the short-term predictability of the chaotic signal and its sensitivity to small interference, the phase space reconstruction of the observation signal of each sensor is carried out. Second, the distributed sensor local linear autoregressive (DS-LLAR) model is constructed to obtain the one-step prediction error of each sensor. Then, we construct a Bayesian risk model and also obtain the corresponding conditional probability density function under each sensor's hypothesis test which firstly needs to fit the distribution of prediction errors according to the parameter estimation. Finally, the fusion optimization algorithm is designed based on the Bayesian fusion criterion, and the optimal decision rule of each sensor and the optimal fusion rule of the fusion center are jointly solved to effectively detect the weak pulse signal in the observation signal. Simulation experiments show that the proposed method which used a distributed sensor combined with a local linear model can effectively detect weak pulse signals from chaotic background.
\end{abstract}

\section{Introduction}

In recent years, with the development of computers, modern signal processing, and sensor technology, multisensor fusion technology has become a research focus in the field of information processing, and it is widely used in military and civil fields [1-3]. Compared with the general signal processing technology, multisensor information fusion technology is a higher-level comprehensive decision-making process based on multisensor measurement results. A multisensor fusion detection system can be arranged in either a centralized or distributed fashion [4]. The fusion center of the centralized system directly performs optimal detection on the observation signals received and transmitted by each sensor, but this system has greater limitations. In the distributed system, the fusion center makes the final judgment on the local judgments made by each sensor on the observation signal; thus, the reliability and survivability of the system are improved while reducing the requirements for communication bandwidth.

At present, distributed information fusion has been paid close attention to by scholars domestically and internationally. An optimal sensor decision rule based on a two-sensor distributed detection problem under fixed fusion rules was proposed by Tenney and Sandell in 1981 [5]. Chair and Varshney proposed an optimization method for fusion rules when the sensor decision rules were fixed [6]. In order to jointly solve the decision rules and fusion rules, a design method of the global optimization detection fusion system was proposed by Reibman and Nolte in 1987 [7]. In order to further improve the detection performance of the fusion system, a series of new optimal fusion algorithms based on covariance, large deviation analysis, least square fusion rules, and Rao test [8-12] of the distributed detection fusion system are proposed. In recent years, many scholars have introduced a neural network [13], Kalman filter [14-16], and (generalized) likelihood ratio (GLRT) [17-20] into sensor systems to realize signal detection in various fields. All the above researches assume that the noise obeys a certain distribution and lack the research on chaotic noise background combined with phase space reconstruction.

The main problem of signal processing under chaotic phenomena is signal detection and recovery, which is widely used in the fields of communication, fault diagnosis, biomedicine, and earthquake monitoring [21-25]. Signal detection 
and recovery in the chaotic background is a method based on a nonlinear system. Scholars domestically and internationally have put forward a series of studies. Xing et al. introduced the support vector machine prediction method of empirical mode decomposition theory with the help of a particle swarm algorithm [26]. Gao et al. used the sensitivity of chaotic systems to parameter perturbation and immunity to noise to construct Duffing $[27,28]$ chaotic oscillator detection; the detection of weak signals is realized by the device [29]. Li et al. proposed a method for measuring the frequency of weak signals under the background of strong noise in the chaotic synchronization system [30]. Some researchers established a series of models to detect and restore signal from chaotic background, such as local linear autoregressive model, dual local linear model, and variable coefficient regression model [31-33]. But the above studies only consider the signal detection of a single sensor and lack research based on the detection of distributed fusion systems.

In order to improve the accuracy and reliability of signal detection, this paper used a coupled local linear model with distributed sensors to perform fusion detection of pulse signals from the background of chaotic noise. Phase space reconstruction (PSR) is firstly used to extract more effective information from the observation sequence. For subsequent distributed fusion, the distributed sensor local linear autoregressive (DS-LLAR) model based on the reconstructed sequence is established to obtain one-step prediction error. Here, we estimate the distribution of one-step prediction error according to least squares. Then, we establish an error risk model based on the Bayes criterion and determine the optimal fusion detection algorithm of the distributed sensor system according to the minimum Bayes risk criterion; that is, the optimal fusion rule and the decision rule are jointly obtained to determine whether the detection signal exists.

The structure of this paper is organized as follows. The second part introduces the distributed fusion system and its hypothesis testing in chaotic background. In the third part, we not only introduce the distributed detection model under chaotic noise and use phase space reconstruction to peel off the chaotic noise but also establish the distributed detection fusion model under the Bayesian criterion. A series of simulation results are reported in the fourth part. The conclusion of this paper is given in the fifth part.

Here is the explanation of the main notation in this article. $y_{i}(t)(i=1,2, \cdots, K)$ is the observation signal of the $i$ th sensor, $\tilde{x}_{i}(t)$ is the chaotic noise signal of the $i$ th sensor, $s(t)$ is the weak pulse signal and is independent of the chaotic noise signal $\tilde{x}_{i}(t), n_{i}(t)$ denotes the white noise with a mean value of 0 , and $x_{i}(t)$ denotes the sum of the chaotic signal $\tilde{x}_{i}(t)$ and white noise $n_{i}(t) . u_{i}(t)$ represents the result of the decision made on the corresponding observation at time $t . y_{i}(t: \tau, m)$ represents the weight of each sensor through the phase space construction of the observation value after adding the time delay order $\tau$ and the embedding dimension $m$, and $e_{i}(t)$ represents the prediction error of each sensor after the DS-LLAR model fitting. The probability of detection is $P_{D, i}$, and the probability of false alarm is $P_{F, i} . P_{D}^{f}$ and $P_{F}^{f}$, respectively, represent the detection probability and false alarm probability of the fusion system. $R_{B}$ is the Bayes risk.

\section{Description of Concepts}

2.1. Distributed Fusion System. The detection fusion system of parallel distributed structure is composed of a fusion center and $K$ detecting sensors (DS), as shown in Figure 1 , where $y_{i}(t)(i=1,2, \cdots, K)$ represents the observation of each sensor on the same target at time $t, u_{i}(t)(i$ $=1,2, \cdots, K)$ represents the result of the decision made on the corresponding observation at time $t$, and $u_{0}(t)$ represents the final decision result made by the fusion center after fusing the decision of each sensor at time $t . u_{i}(t)=1$ means that the judgment result of the $i$ th sensor at time $t$ is that the signal exists, $u_{i}(t)=0$ means that the judgment result of the $i$ th sensor at time $t$ is that the signal does not exist, $u_{0}(t)=0$ means that the decision result of the fusion center at time $t$ is that the signal does not exist, and $u_{0}($ $t)=1$ means that the decision result of the fusion center at time $t$ is that the signal exists. This article assumes that the sensors are independent of each other.

2.2. Distributed Inspection Problem of Chaotic Background. The problem of using multisensor fusion to detect weak pulse signals from chaotic background can be abstracted into the following hypothesis testing problem:

$$
\begin{gathered}
H_{0}: y_{i}(t)=\tilde{x}_{i}(t)+n_{i}(t)=x_{i}(t) \\
H_{1}: y_{i}(t)=\tilde{x}_{i}(t)+s(t)+n_{i}(t)=x_{i}(t)+s(t)
\end{gathered}(i=1,2, \cdots, K),
$$

where $y_{i}(t)$ is the observation signal of the $i$ th sensor, $\tilde{x}_{i}(t)$ is the chaotic noise signal of the ith sensor, $s(t)$ is the weak pulse signal and is independent of the chaotic noise signal $\tilde{x}_{i}(t), n_{i}(t)$ denotes the white noise with a mean value of 0 , and $x_{i}(t)$ denotes the sum of the chaotic signal $\tilde{x}_{i}(t)$ and white noise $n_{i}(t)$.

Since the weak impulse signal $s(t)$ is submerged in the chaotic noise signal $\tilde{x}(t)$, if the hypothesis test is performed directly using equation (1), it is impossible to detect whether the observation signal $y(t)$ contains $s(t)$. Therefore, it is first necessary to remove the interference of the chaotic noise signal $\tilde{x}(t)$ and convert the hypothesis test problem of formula (1) into the following:

$$
\begin{gathered}
H_{0}^{*}: y_{i}(t)-\tilde{x}_{i}(t)=n_{i}(t) \\
H_{1}^{*}: y_{i}(t)-\tilde{x}_{i}(t)=s(t)+n_{i}(t) \quad(i=1,2, \cdots, K) .
\end{gathered}
$$

In the fusion detection problem of distributed parallel architecture, the decision results made by each sensor and the fusion center are a binary value. When $H_{0}^{*}$ is true, the judgment results of the sensor and the fusion center are both 0 , which means that there is no signal. When $H_{1}^{*}$ is true, the judgment results of the sensor and the fusion center are both 1 , which means that there is a 


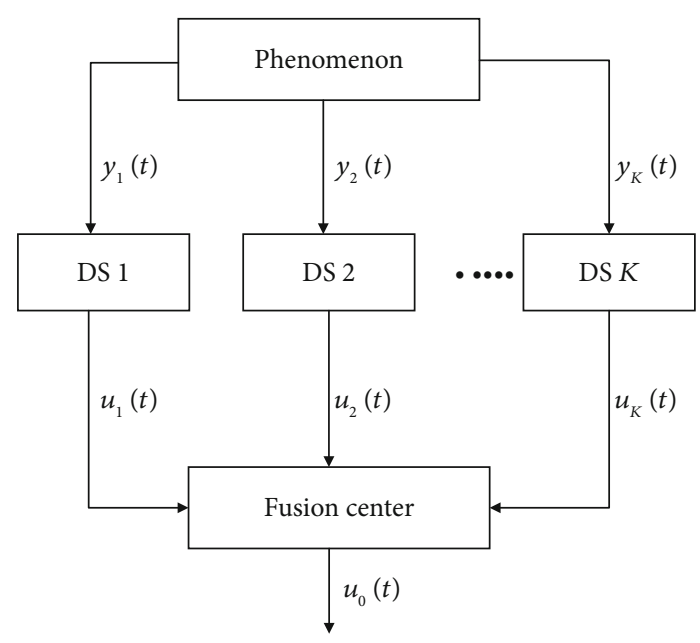

FIgURE 1: General sequence detection fusion system with distributed structure.

signal. Equation (2) is transformed into the following hypothesis testing problem:

$$
\begin{aligned}
& \tilde{H}_{0}: u_{i}=0 \\
& \tilde{H}_{1}: u_{i}=1
\end{aligned} \quad(i=1,2, \cdots, K) .
$$

\section{Distributed Sensor Detection Model}

3.1. Stripping Chaotic Noise. Each sensor needs to reconstruct the phase space of the observation signal and then establish a single step prediction DS-LLAR model for the observation signal to obtain the prediction error.

3.1.1. Phase Space Reconstruction Theory. According to Takens' theorem [34], for $k$ sensors, each observation sequence $\left\{y_{i}(t)\right\}(t=1,2, \cdots, n ; i=1,2, \cdots, k)$. A phase space with $m$ dimension can be constructed by introducing the time delay order $\tau$ and embedding dimension $m$. For the $i$ th sensor, the reconstructed phase space state vector is $\mathbf{Y}_{i}(t)=$ $\left(y_{i}(t), y_{i}(t-\tau), \cdots, y_{i}(t-(m-1) \tau)\right)^{\prime}$, where $t=n_{1}, n_{1}+1$, $\cdots, n ; n_{1}=1+(m-1) \tau$. For any phase point in the reconstructed phase space, we can find a smooth mapping $g$ such that $y_{i}(t+1)=g\left(\mathbf{Y}_{i}(t)\right)\left(t=n_{1}, n_{1}+1, \cdots, n-1\right)$. According to Cao's method [35], the corresponding $\tau$ and $m$ can be obtained.

\subsubsection{DS-LLAR Model.}

$$
\begin{gathered}
y_{i}(t+1) \approx g\left(\mathbf{Y}_{i}(t)\right) \\
g\left(\mathbf{Y}_{i}(t)\right)=\sum_{j=1}^{m} b_{i, j}(t) y_{i}(t-(j-1) \tau)=\boldsymbol{\theta}_{i}^{\prime}(t) \mathbf{Y}_{i}(t)
\end{gathered}
$$

where $\boldsymbol{\theta}_{i}^{\prime}(t)=\left(b_{i, 1}(t), b_{i, 2}(t), \cdots, b_{i, m}(t)\right)^{\prime}$. For the $i$ th sensor, there is a similar evolution law between any phase point in the reconstructed phase space and other phase points around it. The closer the distance is, the greater the degree of evolution similarity is. Therefore, the Euclidean distance is used to determine the $q$ adjacent points, and the Gaussian kernel function is introduced to control the influence of the farther points on the model. The least square estimation is used to estimate $\widehat{\boldsymbol{\theta}}_{i}(t)$.

$$
\begin{aligned}
& \min \sum_{m=1}^{q}\left[y_{i}\left(t_{m}+1\right)-g\left(\mathbf{Y}_{i}\left(t_{\mathrm{m}}\right)\right)\right]^{2} K_{i, h}\left(u_{i, m}\right) \\
& =\min \sum_{m=1}^{q}\left[y_{i}\left(t_{m}+1\right)-\boldsymbol{\theta}_{i}^{\prime}(t) Y_{i}\left(t_{m}\right)\right]^{2} K_{i, h}\left(u_{i, m}\right),
\end{aligned}
$$

where $K_{i, h}\left(u_{i, m}\right)=(1 / h) K_{i}\left(u_{i, m} / h\right), u_{i, m}=d\left(Y_{i, M}\left(t_{m}\right), Y_{i, M}(t)\right.$ ), $\quad K_{i}\left(u_{i, m}\right)=(1 / \sqrt{2 \pi}) \exp \left\{-\left(\left(u_{i, m}\right)^{2} / 2\right)\right\}, \quad m=1,2, \cdots, q$, and $\mathbf{W}_{i}=\operatorname{diag}\left(K_{i, h}\left(u_{i, 1}, u_{i, 2}, \cdots, u_{i, m}\right)\right)_{q \times q}$. The weight between adjacent points is updated by changing the window width $h$.

Set

$$
\mathbf{Y}_{1}=\left[\begin{array}{cccc}
y_{i}\left(t_{1}\right) & y_{i}\left(t_{1}-\tau\right) & \cdots & y_{i}\left(t_{1}-(m-1) \tau\right) \\
y_{i}\left(t_{2}\right) & y_{i}\left(t_{2}-\tau\right) & \cdots & y_{i}\left(t_{2}-(m-1) \tau\right) \\
\vdots & \vdots & \vdots & \vdots \\
y_{i}\left(t_{q}\right) & y_{i}\left(t_{q}-\tau\right) & \cdots & y_{i}\left(t_{q}-(m-1) \tau\right)
\end{array}\right]_{q \times m}
$$

$$
=\left[\begin{array}{c}
\mathbf{X}_{i, M}\left(t_{1}\right) \\
\mathbf{X}_{i, M}\left(t_{2}\right) \\
\vdots \\
\mathbf{X}_{i, M}\left(t_{q}\right)
\end{array}\right]
$$

$\tilde{\mathbf{Y}}_{1}(t)=\left(y_{i}\left(t_{1}+1\right), y_{i}\left(t_{1}+2\right), \cdots, y_{i}\left(t_{i}+1\right)\right)^{\prime}$,

then, generalized degrees of freedom can be expressed as

$$
\begin{aligned}
\widehat{\mathbf{D}}_{i} & \left.=\operatorname{tr}\left(\mathbf{Y}_{i}\left(\mathbf{X}_{i}{ }^{\prime} \mathbf{W}_{i} \mathbf{X}_{i}\right)^{-1}\right) \mathbf{X}_{i}{ }^{\prime} \mathbf{W}_{i}\right), \\
\widehat{\sigma}_{\mathrm{GDF}_{i}}^{2} & =\frac{\left(\tilde{\mathbf{Y}}_{i}-\tilde{\mathbf{Y}} \wedge_{i}\right)^{\prime}\left(\tilde{\mathbf{Y}}_{i}-\widehat{\tilde{\mathbf{Y}}}_{i}\right)}{q-\widehat{\mathbf{D}}_{i}},
\end{aligned}
$$

through minimizing $\widehat{\sigma}_{\mathrm{GDF}_{i}}^{2}$ to obtain the window width $h$ [36].

The local weighted least square method is used to obtain the parameters of formula (6):

$$
\left.\widehat{\boldsymbol{\theta}}_{i}(t)=\left(\mathbf{X}_{i}^{\prime} \mathbf{W}_{i} \mathbf{X}_{i}\right)^{-1} \mathbf{X}_{i}^{\prime} \mathbf{W}_{i}\right) \tilde{\mathbf{Y}}_{i}(t)
$$

By substituting $\widehat{\theta}_{i}(t)$ into equation (5), the single-step prediction $g\left(\mathbf{Y}_{i}(t)\right)$ is obtained, and then, the prediction error $e_{i}(t+1)$ is also obtained:

$$
e_{i}(t+1)=y_{i}(t+1)-g\left(\mathbf{Y}_{i}(t)\right) \text {. }
$$


It is easy to verify that the existence of the prediction error is mainly due to the existence of the weak pulse signal. The hypothesis test problem for the $i$ th sensor is

$$
\begin{aligned}
& H_{0}: y_{i}(t)=\tilde{x}_{i}(t)+n_{i}(t)=x_{i}(t), \\
& H_{1}: y_{i}(t)=\tilde{x}_{i}(t)+s(t)+n_{i}(t)=x_{i}(t)+s(t),
\end{aligned}
$$

where $x_{i}(t)=\tilde{x}_{i}(t)+n_{i}(t)$. When $H_{0}$ is established, perform the phase space reconstruction on the $i$ th sensor observation value $\mathbf{Y}_{i}(t)$; then, there is

$$
\begin{aligned}
& \mathbf{Y}_{i}(t)=\left(y_{i}(t), y_{i}(t-\tau), \cdots, y_{i}(t-(m-1) \tau)\right)^{\prime}=X_{i}(t)+S(t) \\
& \mathbf{X}_{i}(t)=\left(x_{i}(t), x_{i}(t-\tau), \cdots, x_{i}(t-(m-1) \tau)\right)^{\prime} \\
& \mathbf{S}(t)=(s(t), s(t-\tau), \cdots, s(t-(m-1) \tau))^{\prime} .
\end{aligned}
$$

Since local linear autoregression $y_{i}(t+1)=g\left(\mathbf{Y}_{i}(t)\right)=$ $\sum_{j=1}^{m} b_{i, j}(t) y_{i}(t-(j-1) \tau)=\boldsymbol{\theta}_{i}^{\prime}(t) \mathbf{Y}_{i}(t)$, the prediction error can be verified:

$$
\begin{aligned}
e_{i}(t+1)= & y_{i}(t+1)-g\left(\mathbf{Y}_{i}(t)\right) \\
= & x_{i}(t+1)+s(t+1)+n_{i}(t+1)-g\left(\mathbf{X}_{i}(t)\right) \\
& -g(\mathbf{S}(t))-g\left(\mathbf{N}_{i}(t)\right) \\
= & s(t+1)-g(\mathbf{S}(t))+\varepsilon_{i}(t+1),
\end{aligned}
$$

where $\varepsilon_{i}(t+1)=\tilde{x}_{i}(t+1)-g\left(\tilde{\mathbf{X}}_{i}(t)\right)$ and $s(t+1)-g(\mathbf{S}(t))$ are independent of each other. Here, we assume that $\varepsilon_{i}(t)$ does not affect the detection because its independence and large fitting error are produced by $s(t+1)-g(\mathbf{S}(t))$.

3.2. Detection Fusion Model under the Bayesian Criterion. The problem of distributed multisensor detection fusion is to find a set of optimal rules to achieve the best performance of the fusion system. The optimization goal of the optimal distributed detection fusion system is to find a system decision rule that minimizes the Bayes risk of the fusion system.

3.2.1. Chaotic Noise Distributed Fusion System. The distributed fusion system under chaotic noise is shown in Figure 2, where $y_{i}(t)(i=1,2, \cdots, K)$ represents the original observation value of each sensor, $y_{i}(t: \tau, m)(i=1,2, \cdots, K)$ represents the weight of each sensor through the phase space construction of the observation value after adding the time delay order $\tau$ and the embedding dimension $m$, and $\mathrm{e}_{i}(t)(i$ $=1,2, \cdots, K)$ represents the prediction error of each sensor after the DS-LLAR model fitting.

Record the conditional probability density function of the $i$ th sensor observation $e_{i}(t)$ as $f_{i}\left(e_{i}(t) \mid H_{j}\right)(i=1, \cdots, k ; j$ $=0,1)$. The probability of detection is $P_{D, i}$, and the probabil-

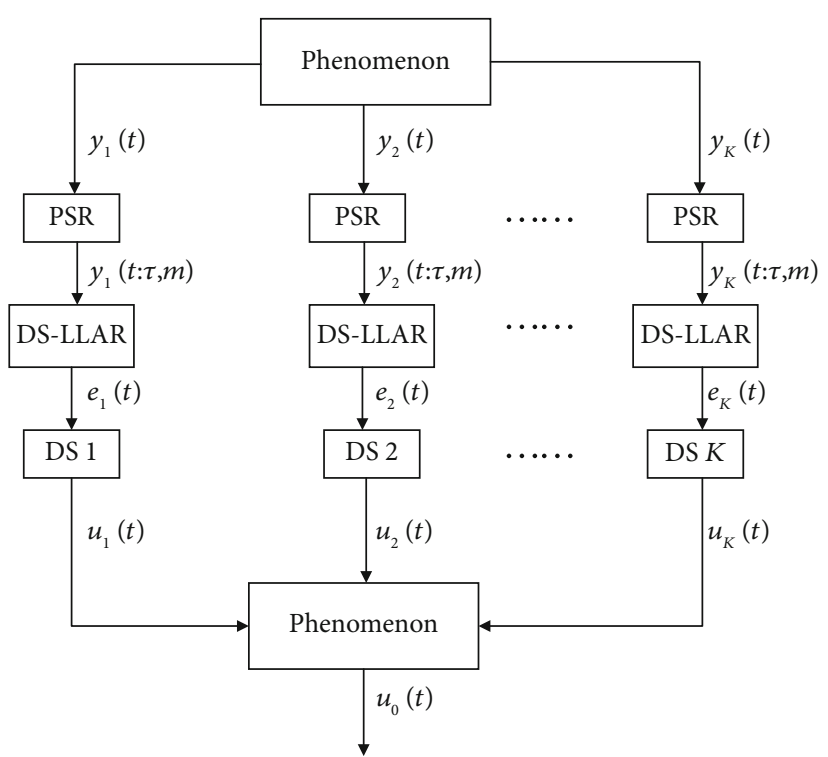

FIgURE 2: Distributed fusion system in chaotic background.

ity of false alarm is $P_{F, i} . P_{D}^{f}$ and $P_{F}^{f}$, respectively, represent the detection probability and false alarm probability of the fusion system. Among them, $f_{i}\left(e_{i}(t) \mid H_{j}\right)$ obeys the normal distribution, and the proof is as follows:

$$
\begin{aligned}
e_{i}(t+1) & =y_{i}(t+1)-g\left(\mathbf{Y}_{i}(t)\right)=y_{i}(t+1)-\boldsymbol{\theta}_{i}^{\prime}(t) \mathbf{Y}_{i}(t) \\
& =y_{i}(t+1)-\sum_{j=1}^{m} b_{i, j}(t) y_{i}(t-(j-1) \tau),
\end{aligned}
$$

where $y_{i}(t+1) \sim N\left(\tilde{x}_{i}(t+1), \sigma^{2}\right)$ and $y_{i}(t-(j-1) \tau) \sim N\left(\tilde{x}_{i}\right.$ $\left.(t-(j-1) \tau), \sigma^{2}\right)$, and they are independent of each other. Therefore,

$e_{i}(t+1) \sim N\left(\left(\tilde{x}_{i}(t+1)-\sum_{j=1}^{m} b_{i, j}(t) \tilde{x}_{i}(t-(j-1) \tau)\right),(1+m) \sigma^{2}\right)$.

Given the prior probability $P_{0}=P\left(H_{0}\right), P_{1}=P\left(H_{1}\right)$, use $C_{n j}$ to denote the cost when $H_{j}$ is true but the judgment is $H_{n}$. The Bayes risk of the fusion system can be expressed as

$$
R_{B}=\sum_{n=0}^{1} \sum_{j=0}^{1} C_{n j} P_{j} P\left(u_{0}=n \mid H_{j}\right)
$$

As a result of $P\left(u_{0}=n \mid H_{1}\right)=\left(P_{D}^{f}\right)^{n}\left(1-P_{D}^{f}\right)^{1-n}, P\left(u_{0}=\right.$ $\left.n \mid H_{0}\right)=\left(P_{F}^{f}\right)^{n}\left(1-P_{F}^{f}\right)^{1-n}$, we simply organize formula (16) to get

$$
R_{B}=\sum_{n=0}^{1} \sum_{j=0}^{1} C_{n j} P_{j} P\left(u_{0}=n \mid H_{j}\right)=C_{F} P_{F}^{f}-C_{D} P_{D}^{f}+C,
$$


where

$$
\left\{\begin{array}{l}
C_{F}=\left(C_{10}-C_{00}\right) P_{0}, \\
C_{D}=\left(C_{01}-C_{11}\right) P_{1}, \\
C=C_{00} P_{0}+C_{01} P_{1} .
\end{array}\right.
$$

Due to $P_{D}^{f}=P\left(u_{0}=1 \mid H_{1}\right), P_{F}^{f}=P\left(u_{0}=1 \mid H_{0}\right)$, we have

$$
\begin{aligned}
& P_{D}^{f}=\sum_{u} P\left(u_{0}=1 \mid \mathbf{u}\right) P\left(\mathbf{u} \mid H_{1}\right), \\
& P_{F}^{f}=\sum_{u} P\left(u_{0}=1 \mid \mathbf{u}\right) P\left(\mathbf{u} \mid H_{0}\right) .
\end{aligned}
$$

Among them, $\boldsymbol{u}$ is a vector composed of the decision results made by each sensor, which only contains two elements of 0 and 1 . So we can get

$$
R_{B}=C+\sum_{u} P\left(u_{0}=1 \mid \mathbf{u}\right)\left[C_{F} P\left(\mathbf{u} \mid H_{0}\right)-C_{D} P\left(\mathbf{u} \mid H_{1}\right)\right] .
$$

It can be seen from equation (17) that the Bayes risk is determined by the decision rules of each sensor and the fusion rules of the fusion center. That is, to minimize the Bayes risk of the fusion center, the optimal fusion rule of the fusion center and the optimal decision rule of each sensor are required.

\subsubsection{Distributed Detection Fusion Algorithm under the Bayes Criterion}

(1) Optimal Fusion Rules. Assuming that the decision rules of each sensor are known, to minimize the Bayes risk $R_{B}$, then the conditional probability should satisfy

$$
P\left(u_{0}=1 \mid \mathbf{u}\right)= \begin{cases}1, & \text { if } C_{F} P\left(\mathbf{u} \mid H_{0}\right)<P\left(\mathbf{u} \mid H_{1}\right), \\ 0, & \text { if } C_{F} P\left(\mathbf{u} \mid H_{0}\right) \geq P\left(\mathbf{u} \mid H_{1}\right) .\end{cases}
$$

That is,

$$
u_{0}= \begin{cases}1, & \text { if } C_{F} P\left(\mathbf{u} \mid H_{0}\right)<P\left(\mathbf{u} \mid H_{1}\right), \\ 0, & \text { if } C_{F} P\left(\mathbf{u} \mid H_{0}\right) \geq P\left(\mathbf{u} \mid H_{1}\right) .\end{cases}
$$

Hence, we can obtain the optimal fusion rule for the fusion center:

$$
\begin{gathered}
H_{1} \\
\frac{P\left(\mathbf{u} \mid H_{1}\right)}{P\left(\mathbf{u} \mid H_{0}\right)}<\frac{\left(C_{10}-C_{00}\right) P_{0}}{\left(C_{01}-C_{11}\right) P_{1}} . \\
H_{0}
\end{gathered}
$$

(1) Optimal Decision Rule. Assume that the fusion rule of the fusion center has been determined; that is, $P\left(u_{0}=1\right.$ | $\mathbf{u})$ is known. If you want to minimize the Bayes risk function value of the fusion system, you want to know the decision rules of each sensor. Obtain the optimal decision rule of the $i$ th sensor, denote $\tilde{\mathbf{u}}_{i}=\left(u_{1}, u_{1}, \cdots, u_{i-1}, u_{i+1}, \cdots\right.$, $u_{K}$ ), which is determined by the false alarm probability of the fusion system $P_{F}^{f}$, and the detection probability $P_{D}^{f}$ can be obtained.

$$
\begin{aligned}
P_{F}^{f}= & \sum_{\tilde{\mathbf{u}}_{i}} P\left(u_{0}=1 \mid \tilde{\mathbf{u}}_{i}, u_{i}=0\right) P\left(\tilde{\mathbf{u}}_{i} \mid H_{0}\right) \\
& +\sum_{\tilde{\mathbf{u}}_{i}} A\left(\tilde{\mathbf{u}}_{i}\right) P\left(\tilde{\mathbf{u}}_{i}, u_{i}=1 \mid H_{0}\right), \\
P_{D}^{f}= & \sum_{\tilde{\mathbf{u}}_{i}} P\left(u_{0}=1 \mid \tilde{\mathbf{u}}_{i}, u_{i}=0\right) P\left(\tilde{\mathbf{u}}_{i} \mid H_{1}\right) \\
& +\sum_{\tilde{\mathbf{u}}_{i}} A\left(\tilde{\mathbf{u}}_{i}\right) P\left(\tilde{\mathbf{u}}_{i}, u_{i}=1 \mid H_{1}\right) .
\end{aligned}
$$

By substituting into equation (17), we can get

$$
\begin{aligned}
R_{B}= & C_{F} \sum_{\tilde{\mathbf{u}}_{i}} A\left(\tilde{\mathbf{u}}_{i}\right) P\left(\tilde{\mathbf{u}}_{i}, u_{i}=1 \mid H_{0}\right) \\
& -C_{D} \sum_{\tilde{\mathbf{u}}_{i}} A\left(\tilde{\mathbf{u}}_{i}\right) P\left(\tilde{\mathbf{u}}_{i}, u_{i}=1 \mid H_{1}\right)+C_{k} .
\end{aligned}
$$

Among them,

$$
\begin{aligned}
A\left(\tilde{\mathbf{u}}_{i}\right)= & {\left[P\left(u_{0}=1 \mid \tilde{\mathbf{u}}_{i}, u_{i}=1\right)-P\left(u_{0}=1 \mid \tilde{\mathbf{u}}_{i}, u_{i}=0\right)\right], } \\
C_{k}= & C_{F} \sum_{\tilde{\mathbf{u}}_{i}} P\left(u_{0}=1 \mid \tilde{\mathbf{u}}_{i}, u_{i}=0\right) P\left(\tilde{\mathbf{u}}_{i} \mid H_{0}\right) \\
& -C_{D} \sum_{\tilde{\mathbf{u}}_{i}} P\left(u_{0}=1 \mid \tilde{\mathbf{u}}_{i}, u_{i}=0\right) P\left(\tilde{\mathbf{u}}_{i} \mid H_{1}\right)+C .
\end{aligned}
$$

Since each sensor makes independent judgments, then

$$
\begin{aligned}
P\left(\tilde{\mathbf{u}}_{i}, u_{i}=n \mid H_{j}\right)= & \int_{e_{i}} P\left(\tilde{\mathbf{u}}_{i} \mid e_{i}, H_{j}\right) P\left(u_{i}=n \mid e_{i}\right) f_{E_{i}} \\
& \cdot\left(e_{i} \mid H_{j}\right) d_{e_{i}}, n, j=0,1, \\
P\left(\tilde{\mathbf{u}}_{i} \mid e_{i}, H_{j}\right)= & P\left(\tilde{\mathbf{u}}_{i} \mid H_{j}\right) .
\end{aligned}
$$

Therefore, the Bayes risk function of the fusion system can be expressed as

$$
\begin{aligned}
R_{B}= & \int_{e_{i}} P\left(u_{i}=1 \mid e_{i}\right)\left\{C_{F} \sum_{\tilde{\mathbf{u}}_{i}} A\left(\tilde{\mathbf{u}}_{i}\right) P\left(\tilde{\mathbf{u}}_{i} \mid H_{0}\right) f_{E_{i}}\left(e_{i} \mid H_{0}\right)\right. \\
& \left.-C_{D} \sum_{\tilde{\mathbf{u}}_{i}} A\left(\tilde{\mathbf{u}}_{i}\right) P\left(\tilde{\mathbf{u}}_{i} \mid H_{1}\right) f_{E_{i}}\left(e_{i} \mid H_{1}\right)\right\} d_{e_{i}}+C_{k} .
\end{aligned}
$$


To minimize the Bayes risk of the fusion system, the conditional probability in (25) needs to satisfy

$$
P\left(u_{i}=1 \mid e_{i}\right)= \begin{cases}1, & \text { if } C_{F} \sum_{\tilde{\mathbf{u}}_{i}} A\left(\tilde{\mathbf{u}}_{i}\right) P\left(\tilde{\mathbf{u}}_{i} \mid H_{0}\right) f_{E_{i}}\left(e_{i} \mid H_{0}\right)<C_{D} \sum_{\tilde{\mathbf{u}}_{i}} A\left(\tilde{\mathbf{u}}_{i}\right) P\left(\tilde{\mathbf{u}}_{i} \mid H_{1}\right) f_{E_{i}}\left(e_{i} \mid H_{1}\right), \\ 0, & \text { others. }\end{cases}
$$

This condition is equivalent to

$$
u_{i}= \begin{cases}1, & \text { if } C_{F} \sum_{\tilde{\mathbf{u}}_{i}} A\left(\tilde{\mathbf{u}}_{i}\right) P\left(\tilde{\mathbf{u}}_{i} \mid H_{0}\right) f_{E_{i}}\left(e_{i} \mid H_{0}\right)<C_{D} \sum_{\tilde{\mathbf{u}}_{i}} A\left(\tilde{\mathbf{u}}_{i}\right) P\left(\tilde{\mathbf{u}}_{i} \mid H_{1}\right) f_{E_{i}}\left(e_{i} \mid H_{1}\right), \\ 0, & \text { others. }\end{cases}
$$

Then, get the optimal decision rule of the ith sensor:

$$
\begin{gathered}
H_{1} \\
\frac{f_{E_{i}}\left(e_{i} \mid H_{1}\right)}{f_{E_{i}}\left(e_{i} \mid H_{0}\right)}<\frac{C_{F} \sum_{\tilde{\mathbf{u}}_{i}} A\left(\tilde{\mathbf{u}}_{i}\right) P\left(\tilde{\mathbf{u}}_{i} \mid H_{0}\right)}{C_{D} \sum_{\tilde{\mathbf{u}}_{i}} A\left(\tilde{\mathbf{u}}_{i}\right) P\left(\tilde{\mathbf{u}}_{i} \mid H_{1}\right)} . \\
H_{0}
\end{gathered}
$$

3.3. Detection Fusion Model under the Bayesian Criterion. We now outline the algorithm.

(Step 1) Reconstruct the phase space of each sensor observation sequence $\left\{y_{i}(t)\right\}(t=1,2, \cdots, n ; i=$ $1,2, \cdots, k)$, and embed the dimension $m$ and the time delay order $\tau$.

(Step 2) Calculate the Euclidean distance, and select $q$ neighboring points of $y_{i}(t)$ in the reconstruction space.

(Step 3) Establish a DS-LLAR model, estimate $\widehat{\boldsymbol{\theta}}_{i}(t)$ using the local weighted least square method, and obtain the forward prediction error $e_{i}(t)$ of each sensor.

(Step 4) Use the least square method to estimate the mean and variance of $e_{i}(t)$, and obtain the conditional probability density function $f_{i}\left(e_{i} \mid H_{j}\right)($ $i=1, \cdots, k ; j=0,1)$ of the $i$ th sensor.

(Step 5) Establish a Bayes risk model $R_{B}$, and obtain the optimal judgment rule and fusion rule based on the Byes risk minimum criterion. The steps to solve the optimal rule are as follows:

(Step 1) The initialization condition is to set the Bayes decision threshold of each sensor as $T_{i}^{0}$ and $P^{0}\left(\mathbf{u} \mid H_{0}\right), P^{0}\left(\mathbf{u} \mid H_{1}\right)$.
(Step 2) Set the loop variable $n$ and the termination control amount $e>0$, and estimate the Bayes fusion criterion $P^{1}\left(u_{0}=1 \mid \mathbf{u}\right)$ and the corresponding $R_{B}^{1}$.

(Step 3) Calculate the new threshold value $T_{i}^{l}, A($ $\left.\tilde{\mathbf{u}}_{i}\right), P\left(\tilde{\mathbf{u}}_{i} \mid H_{0}\right), P\left(\tilde{\mathbf{u}}_{i} \mid H_{1}\right)$, and $P^{l}\left(u_{0}=\right.$ $1 \mid \mathbf{u})$.

(Step 4) Calculate the Bayes risk $R_{B}^{l+1}$ for the $(l+1)$ th iteration.

\section{The Experimental Simulation and Result Analysis}

In order to verify the effectiveness of multisensor detection fusion, four simulation experiments are carried out in this paper. In the experiments in this article, it is assumed that the three sensors are independent of each other and have the same performance. For the three sensors, the Lorenz system is used to generate the chaotic noise background signal. The white noise obeys the normal distribution with the mean value of 0 , and the variance is $0.5,0.6$, and 0.4 , respectively. Use the signal-tonoise ratio $\left(\mathrm{SNR}, \mathrm{SNR}=10 \log \left(\sigma_{s}^{2} /\left(\sigma_{x}^{2}+\sigma_{n}^{2}\right)\right)\right)$ to measure the detection threshold.

The iterative equation of the Lorenz system is as follows:

$$
\left\{\begin{array}{l}
\dot{\eta}=\sigma(y-\eta) \\
\dot{y}=-\eta z+r \eta-y \\
\dot{z}=\eta y-b z
\end{array}\right.
$$

Among them, $\eta, y$, and $z$ are time functions. Set the parameters $\sigma=10, b=8 / 3$, and $r=28$. Supposing the 
TABLE 1: Simulation parameters.

\begin{tabular}{|c|c|c|c|c|c|c|}
\hline \multirow{3}{*}{ Sensor } & \multicolumn{6}{|c|}{ Parameter } \\
\hline & \multicolumn{2}{|c|}{ White noise } & \multirow[b]{2}{*}{ Lorenz system } & \multicolumn{2}{|c|}{ Phase space reconstruction } & \multirow[b]{2}{*}{ Bayesian risk model } \\
\hline & $\begin{array}{l}\text { Mean } \\
\text { value }\end{array}$ & Variance & & $\begin{array}{l}\text { Delay } \\
\text { time } \tau\end{array}$ & $\begin{array}{l}\text { Embedding } \\
\text { dimension } m\end{array}$ & \\
\hline 1 & 0 & 0.5 & \multirow{3}{*}{$\begin{array}{c}\sigma=10, b=8 / 3, r=28, \eta=1, y=1, z=1 \text {, sampling } \\
\text { time } t=0.01\end{array}$} & \multirow{3}{*}{7} & \multirow{3}{*}{6} & \multirow{3}{*}{$\begin{array}{c}c_{00}=c_{11}=0, c_{01}= \\
c_{10}=1\end{array}$} \\
\hline 2 & 0 & 0.6 & & & & \\
\hline 3 & 0 & 0.4 & & & & \\
\hline
\end{tabular}

initial conditions $\eta=1, y=1, z=1$, and sampling time $t$ $=0.01$, using the fourth-order Runge-Kutta method generates 10000 data points, and the first component is taken as chaotic background and recorded as $x(t)$. For the three sensors, the first 3300,2800 , and 3000 points are discarded, respectively, and 4000 continuous time points in the middle are selected as the chaotic background. Use the complex autocorrelation method and Cao's method to determine the delay time $\tau=7$ and the embedding dimension $m=6$ of $y_{i}(t)(i=1,2,3)$. We sort out the parameter settings in Table 1 .

4.1. Experiment 1: Bayes Risk Comparison under Different Signal-to-Noise Ratios. In this experiment, four different signal-to-noise ratios $(-70.0998 \mathrm{~dB},-61.8538 \mathrm{~dB},-58.4526 \mathrm{~dB}$, and $-43.0624 \mathrm{~dB}$ ) are selected to compare the Bayes risk of distributed sensor detection and fusion.

(1) Assume that the pulse signal is a single pulse signal, that is, $s(t)=a_{1} s_{1}(t), a_{1}=5$ :

$$
s_{1}(t)= \begin{cases}1, & t=410,820,1230, \cdots \\ 0, & \text { others }\end{cases}
$$

Generate a time series with a length of 4000 , written as $\{s(t), t=1,2, \cdots, 4000\}$; at this time, $\mathrm{SNR}=-70.0998 \mathrm{~dB}$. The DS-LLAR model is used to fit the signal to obtain the one-step prediction error. Then, the parameter estimation method is used to obtain the conditional probability density function of the prediction error. Finally, a distributed sensor fusion simulation is performed.

(2) Assume that the pulse signal is a superimposed signal of two periodic pulse signals, that is, $s(t)=a_{1} s_{1}(t)$, where $a_{1}=5.5$ :

$$
s_{1}(t)=\left\{\begin{array}{ll}
1, & t=230,460,690, \cdots, \\
0, & \text { others. }
\end{array} .\right.
$$

Generate a time sequence with a length of 4000, marked as $\{s(t), t=1,2, \cdots, 4000\}$; at this time, SNR $=-61.8538 \mathrm{~dB}$. The steps are the same with assumption (1).
(3) Assume that the pulse signal is a superimposed signal of two periodic pulse signals, that is, $s(t)=a_{1} s_{1}(t)+a_{2} s_{2}(t)$, where $a_{1}=5, a_{2}=6$ :

$$
\begin{aligned}
s_{1}(t) & = \begin{cases}1, & t=250,500,750, \cdots, \\
0, & \text { others }\end{cases} \\
s_{2}(t) & = \begin{cases}1, & t=410,820,1230, \cdots, \\
0, & \text { others. }\end{cases}
\end{aligned}
$$

Generate a time sequence with a length of 4000, recorded as $\{s(t), t=1,2, \cdots, 4000\}$; at this time, $\mathrm{SNR}=-58.4526 \mathrm{~dB}$. The steps are the same with assumption (1).

(4) Assume that the pulse signal is a single pulse signal, that is, $s(t)=a_{1} s_{1}(t), a_{1}=6.5$ :

$$
s_{1}(t)= \begin{cases}1, & t=49,98,147, \cdots, \\ 0, & \text { others. }\end{cases}
$$

Generate a time sequence with a length of 4000, recorded as $\{s(t), t=1,2, \cdots, 4000\}$; at this time, $\mathrm{SNR}=-58.4526 \mathrm{~dB}$. The steps are the same with assumption (1). The results are shown in Figure 3.

It can be seen from Figure 3 that the Bayes risk of the optimal fusion system and the sensor changes with the change of the prior probability $p_{0}$ (probability of no signal). In Figure 3(a), when $p_{0}=0.2$, the Bayes risk value of each sensor reaches the maximum, while the Bayes risk of the fusion center is very small. Figures $3(\mathrm{~b})-3(\mathrm{~d})$ are similar to Figure 3(a). The Bayes risk value of each sensor is higher than the Bayes risk value of the fusion center, which indicates that the multisensor detection fusion can significantly reduce the system Bayes risk.

4.2. Experiment 2: Distributed Sensor Fusion ROC Curve under Different Models. In the same SNR, the ROC curve is used to describe and evaluate the accuracy of the signal detection algorithm, which is suitable for the case of two categories (with signal or without signal). The ROC curve is a comprehensive indicator of response sensitivity (true positive rate (TPR)) and specificity (true negative rate (TNR)). The recognition ability of the algorithm in the ROC curve is determined by its position and shape. The area under the curve 


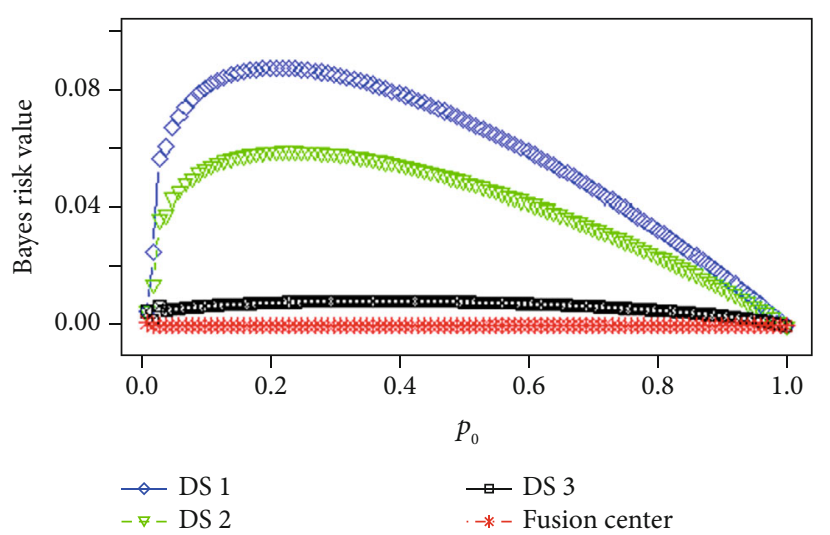

(a)

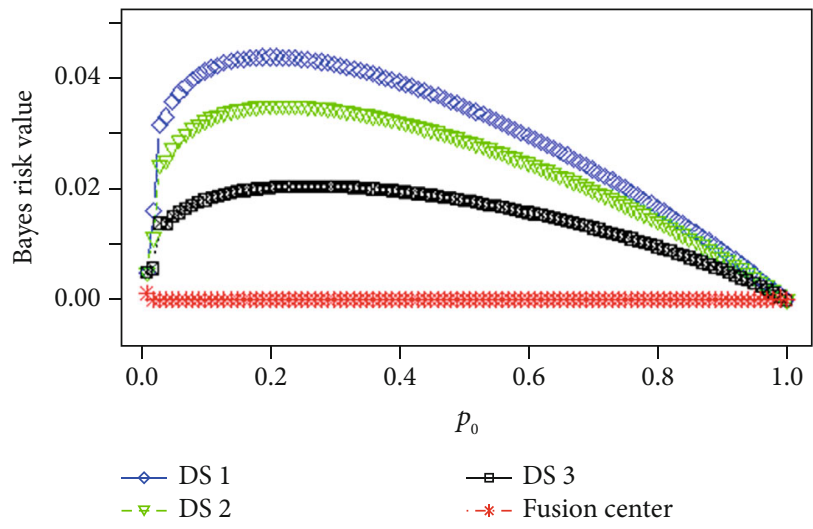

(c)

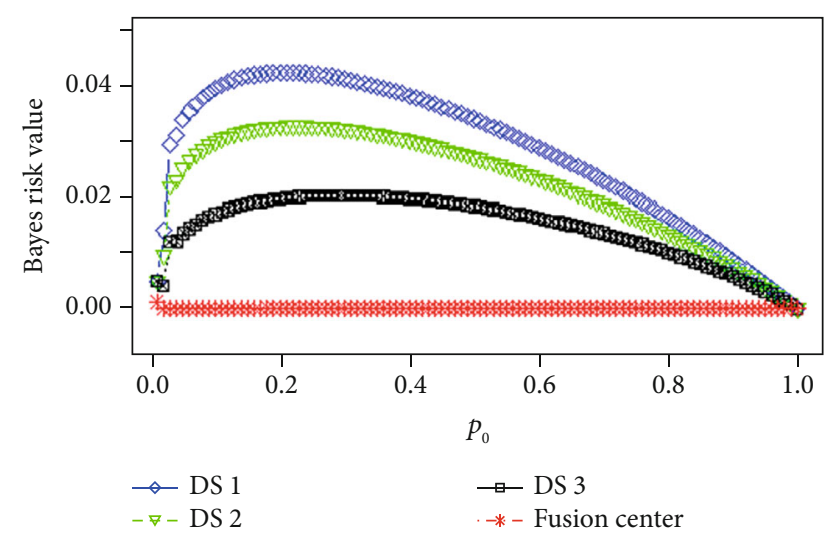

(b)

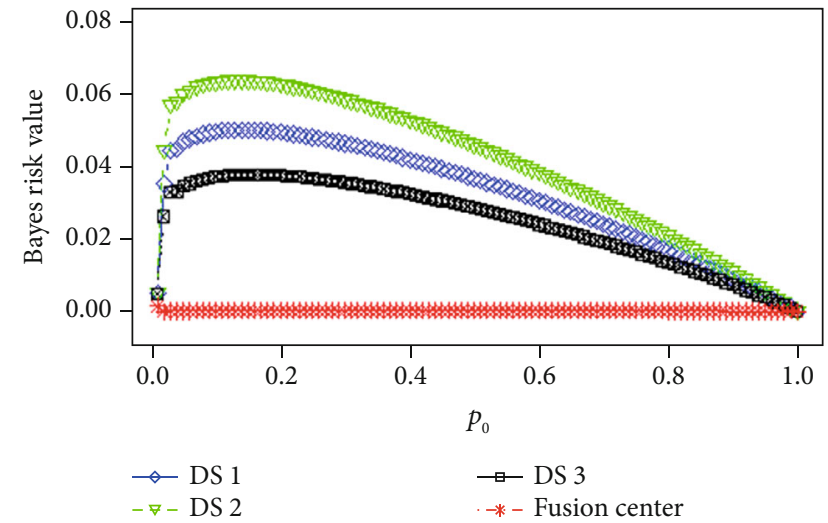

(d)

FIGURE 3: Bayes risk map of three sensors and fusion centers under different SNR: (a) SNR $=-70.0998 \mathrm{~dB}$; (b) SNR $=-61.8538 \mathrm{~dB}$; (c) $\mathrm{SNR}=-58.4526 \mathrm{~dB} ;(\mathrm{d}) \mathrm{SNR}=-43.06236 \mathrm{~dB}$.

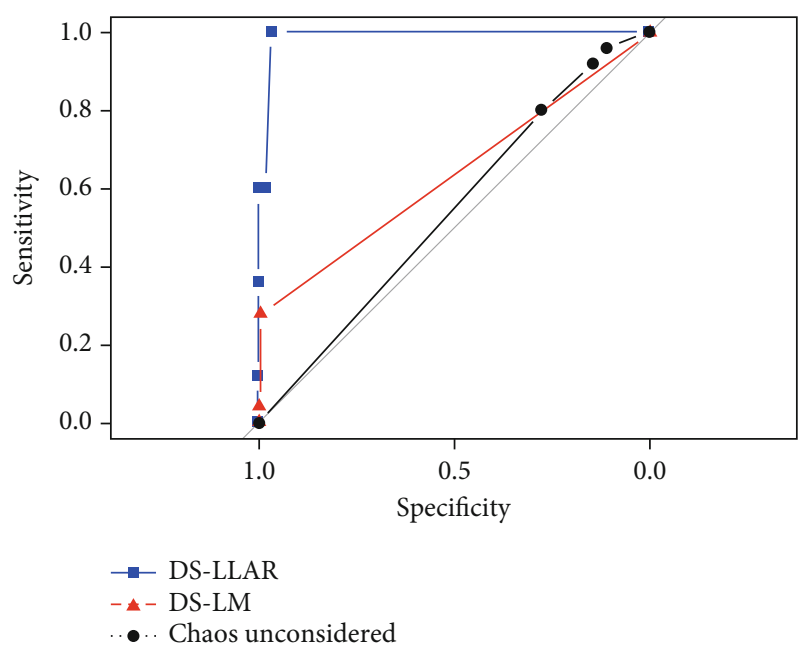

FIGURE 4: Comparison of ROC curves of different models.

(AUC) is used to judge the authenticity of the detection method and reflect the accuracy of the threshold.

Figure 4 shows the three models ((1) DS-LLAR, (2) distributed sensor linear model (DS-LM), and (3) chaos unconsidered) when the signal-to-noise ratio is $-58.4526 \mathrm{~dB}$. The following is ROC curve comparison chart of distributed sensor fusion center.

From the results shown in Table 2, it can be seen that the specificity of the DS-LLAR model is 1 and the sensitivity is 0.967 , indicating that the discrimination accuracy is high and there is no repetitive region between samples. At this time, an AUC of 0.989 further indicates that the threshold accuracy is higher. Although the sensitivity of the DS-LM model is 0.996, the specificity is low, and the AUC value is lower than 0.7 at this time, indicating that the accuracy of the DS-LM model is not high; the specificity when chaos is not considered is 0.800 , but its sensitivity and AUC value are both low, indicating that the accuracy is low without considering chaos. In other words, the DS-LLAR model has the highest diagnostic accuracy.

\subsection{Experiment 3: Comparison of Multisensor Detection} Fusion Performance under Different Models and Different $S N R$. In order to compare the performance of multisensor detection fusion when the SNR and the model are different, this experiment uses four evaluation indicators: accuracy, precision, recall, and F1 score to measure. The accuracy rate can measure the overall accuracy of signal detection. The precision rate and recall rate can evaluate the performance of the 
TABLE 2: Comparison of TPR, TNR, and AUC of different models.

\begin{tabular}{lccc}
\hline Model & TPR & $\begin{array}{c}\text { Index } \\
\text { TNR }\end{array}$ & AUC \\
\hline DS-LLAR & 1.000 & 0.967 & 0.989 \\
DS-LM & 0.280 & 0.996 & 0.638 \\
Chaos unconsidered & 0.800 & 0.275 & 0.543 \\
\hline
\end{tabular}

model. The precision rate indicates the proportion of targets that are predicted to belong to the category in a certain category, and the recall rate indicates the proportion of all correctly identified targets to all the targets that should be identified. The F1 score is a comprehensive indicator of precision and recall, which can punish extreme situations.

$$
\begin{aligned}
\text { Accuracy } & =\frac{\mathrm{TP}+\mathrm{TN}}{\mathrm{TP}+\mathrm{FP}+\mathrm{TN}+\mathrm{FN}}, \\
\text { Precision } & =\frac{\mathrm{TP}}{\mathrm{TP}+\mathrm{FP}}, \\
\text { Recall } & =\frac{\mathrm{TP}}{\mathrm{TP}+\mathrm{FN}}, \\
\text { F1 score } & =\frac{\text { Precision } * \text { Recall } * 2}{\text { Precision }+ \text { Recall }} .
\end{aligned}
$$

In the formula, $\mathrm{TP}$ is the number of samples with signal detected, FP is the number of samples with signal but not detected with signal, FN is the number of samples with no signal detected by mistake, and TN is the number of samples with no signal and detected no signal.

The results of Tables 3-6 show that under the four SNR, the accuracy of the multisensor detection fusion after fitting by the local linear model and the linear model is higher than $98 \%$. When the SNR is $-70.0998 \mathrm{~dB}$ and $-61.8538 \mathrm{~dB}$, the detection accuracy, recall rate, and F1 score of DS-LLAR fitting are higher than those of DS-LM fitting, and at this time, the linear model did not detect the correct signal. When the SNR is $-58.4526 \mathrm{~dB}$, the accuracy of the detection after DSLLAR fitting is lower than that of DS-LM, and the recall rate (0.04) and F1 score (0.0769) of DS-LM are far lower than the recall rate (0.6) and F1 score (0.6122) of DS-LLAR. When the SNR is $-43.06236 \mathrm{~dB}$, the detection accuracy of DS-LLAR is about $8 \%$ lower than that of DS-LM, but the recall rate and F1 value are still higher than those of DS-LM. Among them, the recall rate when the SNR is $-43.0624 \mathrm{~dB}$ is relatively lower than the F1 value. However, when signal detection is performed directly without considering chaos, the recall rate is high, but the accuracy, precision, and F1 score evaluation indicators are low; hence, the detection effect is extremely poor. In conclusion, the performance of multisensor detection fusion after DS-LLAR fitting is the best.

4.4. Experiment 4: The Computational Complexity of the Two Methods Is Compared. The sample size is set as an arithmetic sequence from 10000 to 910000 , with a tolerance of 100000 . The median time of 100 simulations for each sample size is tested as the running time standard of the model under the
TABLE 3: $\mathrm{SNR}=-70.0998 \mathrm{~dB}$ detection effect under different models.

\begin{tabular}{lcccc}
\hline \multirow{2}{*}{ Model } & \multicolumn{4}{c}{ Index } \\
& Accuracy & Precision & Recall & F1 score \\
\hline DS-LLAR & 0.9982 & 0.5833 & 0.7778 & 0.6667 \\
DS-LM & 0.9977 & Nan & 0 & Nan \\
Chaos unconsidered & 0.1062 & 0.0020 & 0.7778 & 0.0039 \\
\hline
\end{tabular}

Note: Nan is generated when the denominator is 0 , because the system does not detect the correct signal.

TABLE 4: $\mathrm{SNR}=-61.8538 \mathrm{~dB}$ detection effect under different models.

\begin{tabular}{lcccc}
\hline \multirow{2}{*}{ Model } & \multicolumn{4}{c}{ Index } \\
& Accuracy & Precision & Recall & F1 score \\
\hline DS-LLAR & 0.9965 & 0.5883 & 0.5883 & 0.5883 \\
DS-LM & 0.9957 & Nan & 0.0000 & Nan \\
Chaos unconsidered & 0.1118 & 0.0048 & 1.0000 & 0.0096 \\
\hline
\end{tabular}

TABle 5: $\mathrm{SNR}=-58.4526 \mathrm{~dB}$ detection effect under different models.

\begin{tabular}{lcccc}
\hline \multirow{2}{*}{ Model } & \multicolumn{4}{c}{ Index } \\
& Accuracy & Precision & Recall & F1 score \\
\hline DS-LLAR & 0.9952 & 0.6250 & 0.6000 & 0.6123 \\
DS-LM & 0.9939 & 1.0000 & 0.0400 & 0.0769 \\
Chaos unconsidered & 0.1118 & 0.0062 & 0.8800 & 0.0123 \\
\hline
\end{tabular}

TABLE 6: $\mathrm{SNR}=-43.0624 \mathrm{~dB}$ detection effect under different models.

\begin{tabular}{lcccc}
\hline \multirow{2}{*}{ Model } & \multicolumn{4}{c}{ Index } \\
& Accuracy & Precision & Recall & F1 score \\
\hline DS-LLAR & 0.9836 & 0.8077 & 0.2593 & 0.3925 \\
DS-LM & 0.9813 & 0.8889 & 0.0988 & 0.1778 \\
Chaos unconsidered & 0.1294 & 0.0210 & 0.9136 & 0.0411 \\
\hline
\end{tabular}

sample size. The computational complexity of the two methods is $o(n)$. According to Table 7 and Figure 5, comparing the computational time of the two methods, the computational time increases linearly with the increase in sample size, and the order of magnitude and growth speed of the linear model is faster than that of the local linear model. Therefore, the local linear model is better.

\section{Conclusions}

Combined with the short-term predictability of chaotic time series and the sensitivity to small disturbances, based on the DS-LLAR algorithm, this paper proposes a distributed sensor fusion method to detect impulse signals under the background of chaotic noise. From the experimental results, we can draw the following conclusions: (1) The DS-LLAR model 
TABLE 7: Complexity (unit: seconds).

\begin{tabular}{lcc}
\hline Sample & DS-LLAR & Model \\
\hline 1 & 0.00100 & DS-LM \\
2 & 0.00200 & 0.00200 \\
3 & 0.00251 & 0.00800 \\
4 & 0.00400 & 0.01300 \\
5 & 0.00500 & 0.02002 \\
6 & 0.00600 & 0.03000 \\
7 & 0.00700 & 0.03600 \\
8 & 0.00800 & 0.04400 \\
9 & 0.00950 & 0.05100 \\
10 & 0.01000 & 0.05800 \\
\hline
\end{tabular}

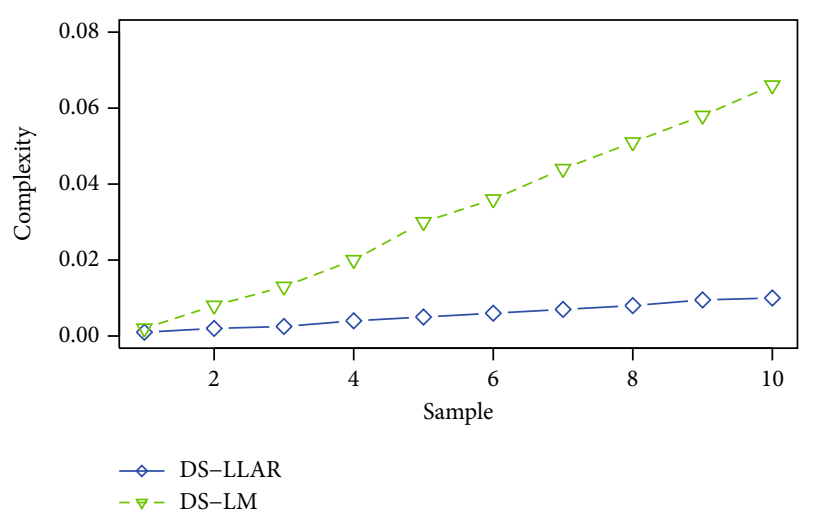

Figure 5: Complexity.

can strip chaotic noise well, and when combined with the distributed sensor fusion system, the Bayes risk of the system can be minimized. (2) According to the results of the ROC curve, the accuracy of the fusion center detection algorithm under the DS-LLAR model is the highest. (3) Compared with other models under different SNR, it is more intuitive to show that the prediction error of the DS-LLAR model after fitting can make the detection performance of the distributed sensor system reach the best. (4) The computational complexity of DS-LLAR is better. In the future, we will continue to explore the influence of the prediction error of the DSLLAR model on the subsequent construction of the detection model, so as to achieve a lower SNR threshold. We will continue to explore the application of the DS-LLAR model in signal detection under different chaotic backgrounds and compare it with the machine learning method, so as to extend this model to other signal detection.

\section{Data Availability}

No data were used to support this study.

\section{Conflicts of Interest}

The authors declare that there are no conflicts of interest regarding the publication of this paper.

\section{Acknowledgments}

This work was supported by the Chongqing Natural Science Foundation of China (Grant nos. cstc2018jcyjAX0464 and cstc2019jcyj-msxmX0491) and Chongqing Postgraduate Research and Innovation Project Funding (Project No. clgycx 20203143).

\section{References}

[1] P. Ma, F. Lu, H. L. Du, R. Wang, and C. L. Niu, "Theory and application of multi-sensor information fusion," Control Engineering of China, vol. 13, no. 1, pp. 48-51, 2006.

[2] Q. Pan, Z. F. Wang, Y. Liang, F. Yang, and Z. G. Liu, "Basic methods and progress of information fusion (II)," Control Theory \& Applications, vol. 29, no. 10, pp. 1233-1244, 2012.

[3] G. Psuj, "Multi-sensor data integration using deep learning for characterization of defects in steel elements," Sensors, vol. 18, no. 2, p. 292, 2018.

[4] H. Chongzhao, H. Zhu, and D. Shengli, Multi-Source Information Fusion, Tsinghua University Press, Second edition, 2010.

[5] R. R. Tenney and N. R. J. Sandell, "Detection with distributed sensors," IEEE Transactions on Aerospace and Electronic Systems, vol. AES-17, no. 4, pp. 501-510, 1981.

[6] Z. Chair and P. K. Varshney, "Optimal data fusion in multiple sensor detection systems," IEEE Transactions on Aerospace and ElectronicSystems, vol. AES-22, no. 1, pp. 98-101, 1986.

[7] A. R. Reibman and L. W. Nolte, "Optimal detection and performance of distributed sensor systems," IEEE Transactions on Aerospace and Electronic Systems, vol. AES-23, no. 1, pp. 24-30, 1987.

[8] H. F. Han, W. H. Zhou, and G. H. Chen, "Decentralized multisensor management algorithm based on covariance control for target tracking," Modern Radar, vol. 1, 2007.

[9] D. Li, S. Kar, F. E. Alsaadi, and S. Cui, "Distributed Bayesian quickest change detection in sensor networks via two-layer large deviation analysis," IEEE Internet of Things Journal, vol. 99, p. 1, 2018.

[10] Y. Z. Cai, H. Y. Chen, and C. L. Wan, "Distributed dynamic state estimation for power systems based on least square estimation fusion," Automation of Electric Power Systems, vol. 40, no. 8, pp. 29-35+113, 2016.

[11] S. Zhu, C. Chen, J. Xu, X. Guan, L. Xie, and K. H. Johansson, "Mitigating quantization effects on distributed sensor fusion: a least squares approach," IEEE Transactions on Signal Processing, vol. 66, no. 13, pp. 3459-3474, 2018.

[12] B. Chen, R. Jiang, T. Kasetkasem, and P. K. Varshney, "Channel aware decision fusion in wireless sensor networks," IEEE Transactions on Signal Processing, vol. 52, no. 12, pp. 34543458, 2004.

[13] H. H. Jia and J. Q. Liu, "Reliability analysis of distributed sensor network based on back propagation neural network," Journal of the China Railway Society, vol. 5, pp. 80-83, 2002.

[14] C. Li, H. Dong, J. Li, and F. Wang, "Distributed Kalman filtering for sensor network with balanced topology," Systems \& Control Letters, vol. 131, 2019.

[15] Z. Z. Wu, M. Y. Fu, Y. Xu, and R. Q. Lu, “A distributed Kalman filtering algorithm with fast finite-time convergence for sensor networks," Automatica, vol. 95, pp. 63-72, 2018. 
[16] Y. Zhang and Y. P. Tian, "A fully distributed weight design approach to consensus Kalman filtering for sensor networks," Automatica, vol. 104, pp. 34-40, 2019.

[17] R. Niu and P. K. Varshney, "Performance analysis of distributed detection in a random sensor field," IEEE Transactions on Signal Processing, vol. 56, no. 1, pp. 339-349, 2007.

[18] D. Ciuonzo and P. S. Rossi, "Distributed detection of a noncooperative target via generalized locally- optimum approaches," Information Fusion, vol. 36, pp. 261-274, 2017.

[19] X. Cheng, D. Ciuonzo, and P. S. Rossi, "Multibit decentralized detection through fusing smart and dumb sensors based on Rao test," IEEE Transactions on Aerospace and Electronic Systems, vol. 56, no. 2, pp. 1391-1405, 2020.

[20] D. Ciuonzo, S. H. Javadi, A. Mohammadi, and P. S. Rossi, "Bandwidth-constrained decentralized detection of an unknown vector signal via multisensor fusion," IEEE Transactions on Signal and Information Processing Over Networks, vol. 6 , pp. 744-758, 2020.

[21] J. Eckmann and D. Ruelle, "Ergodic theory of chaos and strange attractors," Review of Modern Physics, vol. 57, no. 3, pp. 1115-1115, 2008.

[22] Y. B. Wu, M. Zhu, W. Q. Zhu, and Z. P. Xing, "Signal processing algorithm for noncoherent underwater acoustic communication approaching channel capacity," Acta Acustica, vol. 40, no. 1, pp. 117-123, 2015.

[23] V. Demjanenko and R. A. Valtin, "A noninvasive diagnostic instrument for power circuit breakers," IEEE Transactions on Power Delivery, vol. 7, no. 2, pp. 656-663, 1992.

[24] L. Sun, M. Shen, F. H. Y. Chan, and P. J. Beadle, "A novel method of time-frequency representation and its application to biomedical signal processing," in Proceedings 7th International Conference on Signal Processing, 2004. Proceedings. ICSP '04. 2004, pp. 236-239, Beijing, China, August-September 2004.

[25] P. J. Chung, M. L. Jost, and J. F. BHme, "Estimation of seismicwave parameters and signal detection using maximumlikelihood methods," Computers \& Geosciences, vol. 27, no. 2, pp. 147-156, 2001.

[26] H. Y. Xing, Q. Zhang, and W. Xu, "Hybrid algorithm for weak signal detection in chaotic sea clutter," Acta Physica Sinica, vol. 64 , no. 4, pp. 52-58, 2015.

[27] H. Chen and P. K. Varshney, "Theory of the stochastic resonance effect in signal detection-part II: variable detectors," IEEE Transactions on Signal Processing, vol. 56, no. 10, pp. 5031-5041, 2008.

[28] H. Chen, P. K. Varshney, S. M. Kay, and J. H. Michels, “Theory of the stochastic resonance effect in signal detection: part I-fixed detectors," IEEE transactions on Signal Processing, vol. 55, no. 7, pp. 3172-3184, 2007.

[29] S. L. Gao, S. C. Zhong, K. Wei, and H. Ma, "Weak signal detection based on chaos and stochastic resonance," Acta Physica Sinica, vol. 61, no. 18, pp. 45-51, 2012.

[30] G. Z. Li, N. L. Tan, S. Q. Su, and C. Zhang, "Unknown frequency weak signal detection based on Lorenz chaotic synchronization system," Journal of Vibration and Shock, vol. 38, no. 5, pp. 155-161, 2019.

[31] L. Y. Su, Z. H. Sun, J. Wang, and L. M. Yang, "Detection and recovery of weak pulse signal in chaotic noise background," Acta Physica Sinica, vol. 66, no. 9, pp. 29-38, 2017.

[32] L. Y. Su and C. L. Li, "Extracting narrow-band signal from a chaotic background with LLVCR," Wireless Personal Commu- nications: An International Journal, vol. 96, pp. 1907-1927, 2017.

[33] L. Y. Su and C. L. Li, "Extracting harmonic signal from a chaotic background with local linear model," Mechanical Systems \& Signal Processing, vol. 84, pp. 499-515, 2017.

[34] F. Takens, "Detecting strange attractors in turbulence, in dynamical systems and turbulence, Warwick," Lecture Notes in Mathematics, vol. 1981, p. 898, 1980.

[35] L. Cao, "Practical method for determining the minimum embedding dimension of a scalar time series," Physica D: Nonlinear Phenomera, vol. 110, no. 1-2, pp. 43-50, 1997.

[36] D. She and X. Yang, "A new adaptive local linear prediction method and its application in hydrological time series," Mathematical Problems in Engineering, vol. 2010, Article ID 205438, 15 pages, 2010. 